Snežana MILINKOVIĆ*

Università di Belgrado

\title{
«IL BUON GOVERNO DELLA CASA». UNA LETTERA ECONOMICA DI LODOVICO BECCADELLI
}

Parole chiave: Lodovico Beccadelli, lettere, autobiografia, utile, onore.

«Il verso in questione, oltre a marcare l'endiadi sulla quale si regge, lascia intravedere come l'intento di Dante...». È difficile sottrarsi alla tentazione di immergersi nelle acque care a Mirka Zogović e di provare a seguirne la corrente, facendo riaffiorare le sue parole, che ancora riecheggiano tra le pareti dell'aula in cui era solita tenere lezione. E ancor più difficile è mitigare il perdurante effetto che il timbro e la modulazione della voce producevano, e che esortava a mettersi in sintonia con il senso e la portata del messaggio che vi erano riposti. Ma occorre, d'altra parte, ammettere che non sempre è consigliabile abbandonarsi alla forza della suggestione e che talvolta è anzi d'obbligo resisterle, specie allorché - come in questo caso - ai buoni propositi non viene a corrispondere la richiesta, indispensabile dotazione che servirebbe per assecondarli.

Si giustifica così la pur dolente rinuncia ad ogni pretesa di cimento con le elevatezze di dantesco respiro e il conseguente ripiego in direzione dei piu accessibili e rassicuranti lidi della sponda orientale dell'Adriatico, con l'incoraggiante prospettiva, se non altro, di poter fornire un modesto contributo ai cultori della materia ragusea. La lettera, ad oggi inedita, che qui presentiamo - e che riportiamo nella sua interezza - è stata scritta da Lodovico Beccadelli nel 1559, nel corso dell'ultimo anno di permanenza nella città dalmata. Indirizzata al conte Ludovico Tedesco, rientra nel novero di quelle lettere che il Beccadelli, al rientro in patria, avrebbe raccolto e

*snezana.milinkovic@fil.bg.ac.rs 
disposto cronologicamente in appositi quaderni, in vista di un'auspicata - e regolarmente rinviata - pubblicazione, in realtà mai avvenuta. ${ }^{1}$

Colto uomo di Chiesa, umanista dai poliedrici interessi, devoto e fedele servitore di maestri e signori, che «quanto è più onorato et inalzato, tanto più s'abbassa et s'humilia», il Beccadelli si era guadagnato una certa riputazione anche in virtù di notevoli ed apprezzate biografie, che spaziavano da Pietro Bembo al cardinale Contarini. ${ }^{2}$ E tuttavia, se ammirata ed elogiata era la diligenza con cui affrontava gli impegni d'ufficio - quasi un'incarnazione della modestia e della costanza che si esigevano da un funzionario di quel livello - al Beccadelli va soprattutto riconosciuto il merito di essere stato un attento e scrupoloso osservatore degli accadimenti del tempo, che meticolosamente e con grande finezza registrava negli appunti che prendeva e che consegnava alle lettere che spediva ad amici, parenti, conoscenti. La sua collezione di «carte messaggere», prodighe di ponderate valutazioni e di preziosi consigli, viene così ad assumere il ruolo di un'impagabile testimonianza, non solo delle vicende e delle pulsioni ideali e culturali che hanno caratterizzato l'epoca in cui è vissuto - e, per tale via, della «retorica e dei modelli di comunicazione epistolare» cui la loro narrazione era piegata - ma pure delle attese, delle aspirazioni, delle preoccupazioni che un uomo nella sua posizione era chiamato ad affrontare. ${ }^{3}$

${ }^{1}$ Sulle lettere del periodo raguseo conservate nell'Archivio Beccadelli della Biblioteca Palatina di Parma si rinvia all'introduzione al volume edito dall'Accademia: S. Milinković, Predgovor, in Prepiska Lodovika Bekadelija, nadbiskupa dubrovačkog (1555-1560), a cura di S. Milinković - N. Stipčević, Posebna izdanja, DCLXXXII, Odeljenje jezika i književnosti, 59, Beograd, Srpska akademija nauka i umetnosti, 2016, pp. 7-28. Sull'Archivio Beccadelli v. M. C. TARSI, L'epistolario di Lodovico Beccadelli. Con un'appendice sui carteggi beccadelliani dispersi, in Epistolari dal Due al Seicento: modelli, questioni ecdotiche, edizioni, cantieri aperti, a cura di C. Berra, P. Borsa, M. Comelli e S. Martinelli Tempesta, Milano, Università degli Studi, 2018, pp. 316-376.

${ }^{2}$ Sono ormai numerosi i contributi dedicati a singoli aspetti dell'attività di L. Beccadelli, ma restano insostituibili i lavori di G. Fragnito, Memoria individuale e costruzione biografica. Beccadelli, Della Casa, Vettori alle origini di un mito, Urbino, Argalia Editore, 1978 (si veda, a p. 61, il segmento della lettera di Galasso Ariosto del 6 giugno 1545, da cui è tratta la citazione), e della stessa G. FraGnito, In museo e in villa. Saggi sul Rinascimento perduto, Venezia, Arsenale Editrice, 1988.

${ }^{3}$ D'obbligo il richiamo all'ormai classico A. Quondam, Dal "formulario" al "formulario": cento anni di "libri di lettere", in Le "carte messaggere". Retorica e modelli di comunicazione epistolare: per un indice di libri di lettere del Cinquecento, a cura di A. Quondam, Roma, Bulzoni, 1981, pp. 13-156. Sul rapporto tra il libro di lettere e l'autobiografia cfr. G. GENOVESE, La lettera oltre il genere. Il libro delle lettere, dall'Aretino al Doni, e le origini dell'autobiografia moderna, Padova, Antenore, 2009. 
Un'autobiografia, insomma. È questo, in fondo, il risultato che si ricava dallo scorrimento delle lettere del Beccadelli. Che fornivano, con il loro andamento, frutto di una ben soppesata selezione, l'angolazione con la quale, nel suo disegno, i posteri avrebbero dovuto coglierlo. Lo ribadisce pure la lettera qui allegata: siamo agli sgoccioli dell'esperienza ragusea, vissuta inizialamente come una punizione, ma il Beccadelli mostra di non serbare rancore e di dover persino ringraziare la Provvidenza, per averlo messo con le spalle al muro e averlo costretto a reggere la prova. Fa capire di esserne uscito rafforzato, di aver saputo reagire alle avversità. È con tono paterno, infatti («com'a mio dilettissimo figliolo»), che si rivolge al «giovane» interlocutore, «allevato gentilhomo», per ricordargli quale sia la condotta da assumere per «il buon governo della Casa», a beneficio del «commun patrone», il suo vecchio discepolo, il cardinale Ranuccio Farnese (che di lì a poco si sarebbe ritrovato sulla soglia dello scranno pontifico, riuscendo comunque ad imporsi, assieme al fratello Alessandro, nell'elezione di Pio $\mathrm{IV}$, poi decisivo anche per le sorti dello stesso Beccadelli). ${ }^{4}$ Non mancano, ovviamente, i precetti di ordine generale, studiati ed approfonditi sì sulla scorta di meditate letture («Cicerone, Aristotile»), ma ancor più in virtù di una prolungata e proficua esperienza personale (lo scrivente, dopotutto, ha «servito et conosciuto domesticamente li Reverendissimi Contarino et Polo»). Perché non si tratta, semplicemente di provvedere ad un'oculata ed equa gestione di affari e sottoposti (assicurandosi, per i primi, che «alcun ministro non facesse incetta coi danari del patrone», ed evitando, nel caso degli altri, appoggi e vantaggi «se non secondo li meriti et buoni portamenti»), bensì di agire sempre con la massima circospezione, tenendo ben presente «l'avertenza», valida per qualsiasi genere di «negotio», che «l'honore... va sempre innanzi all'utile», sebbene molti non vi badino ed «anzi solo reputano honesto quello ch'è utile».

Le istruzioni impartite al protetto del cardinale, che ne ha dato un «honorato iuditio», vengono così ad assumere il profilo di un vero e proprio trattatello morale («forse ho detto troppo», se «questa lettera havesse ad andare in mano d'altri, io sarei stato più ristrettoso et modesto»), le cui conclusioni sembrano, in fondo, risolversi nell'analogia presa in prestito dagli antichi, secondo la quale le principali virtù che si richiedono, la prudentia e la sapientia, sono disposte gerarchicamente, come «il maior domo col patrone»: suo compito sarà, pertanto, quello d'affaticarsi e travagliare «perché il signore possa più commodamente attendere a cose maggiori». Ma è importante sottolineare come in tali istruzioni, che un

${ }^{4}$ Più che sufficienti, in proposito, le indicazioni fornite dal Dizionario Biografico degli Italiani, sotto la voce Ranuccio Farnese. 
corteggiano minore, di famiglia costumata, era chiamato ad assimilare, si ravvisino le linee guida di una precettistica comportamentale oramai largamente acquisita, che presumeva di poter regolare ogni aspetto della vita sociale attraverso la pratica del negozio: stima e riputazione, danno e vergogna erano oggetto di scambio e di mercato al pari di ogni altro bene o risorsa materiale. ${ }^{5}$ Sulla scia di un (mai menzionato) F. Guicciardini o di un B. Castiglione, anche il disincantato Beccadelli, che ben sapeva "come vanno le cose del mondo", aveva maturato la convinzione che la norma morale dovesse essere «distintiva e relativa», frutto della convenienzia, secolarizzata e mondana, e che l'uso virtuoso della parola, che contemplava anche strategie di simulazione e di dissimulazione, andava a tutto profitto dell'utile e dell'honore. ${ }^{6}$

Era, a ben vedere, il sistema etico che l'Italia del Quattrocento aveva elaborato e che il primo Cinquecento aveva portato a piena consacrazione. Per spodestarlo si sarebbe dovuto attendere non poco: a pensarci sarebbe stato Immanuel Kant.

\section{Al Conte Ludovico Todesco a Roma, alli 25. di Maggio 1559.}

Della lettera di V. S. di 22. d'Aprile ricevuta alli 17. di questo ho preso grandissimo piacere, sì per vedere ch'ella di me tiene amorevole memoria, il che stimo assai, et per haver inteso l'honorato iuditio che di lei ha fatto Monsignor Illustrissimo commun patrone con darli il governo della sua Casa, et quanto è maggiore tanto più mostra quanto la stimi. Io fo più conto signor mio di questo iuditio che se v'havesse donato nella patria vostra cinquecento fiorini d'entrata, perché quello si potria dire che procedesse dall'amore che solamente alla vostra bontà portasse, dove in questo mostra non solo di amarvi, ma stimarvi assai, con non poca riputatione di V. S. et tanto più quanto credo che già molti anni non si sia visto in Roma a un giovane dell'età sua dare simile carico. Ma la sua bontà et virtù meritano questo et più et gli apriranno la strada a maggior grado. Quanto alla parte che mi ricerca ch'io li dia alcun ricordo per il buon governo della Casa, non mi accaderia entrare in questo ch'ella da se et col cenno del patrone, ch'è savio, potrà benissimo governarsi, pur per non parer che non desideri di cimpiacerla et per ragionare alquanto più seco, li ricordarò com'a mio dilettissimo figliolo ch'ella la prima cosa non pigli affettione o parte in Casa, se non secondo li meriti et buoni portamenti delle persone, habbiali tutti come fratelli et cerchi giovare a tutti in quello per lei si potrà et tocca all'uffitio

${ }^{5}$ Cfr. A. Quondam, Forma del vivere. L'etica del gentiluomo e i moralisti italiani, Bologna, il Mulino, 2010, pp. 512-515.

${ }^{6}$ Cfr. A. Quondam, Forma del vivere. L'etica del gentiluomo e i moralisti italiani, cit., pp. 549-559. 
suo; sopratutto avertisca che li ministri suoi, siano leali et di buona vita, perché questi tali sono come le dita della mano et però bisogna tenerli netti, et diligenti negli uffiti loro che così terranno la casa contenta et a voi faranno honore.

Ricordarovi anchora ben che sia soverchio con voi che sete nato et allevato gentilhomo, cioè che guardiate che qualche volta alcun ministro non facesse incetta coi danari del patrone, che tornasse a suo pro et non della Casa, perché questi tali atti sono sordidi et puzzano di furto. Tutti gli avantaggi che si ponno trovare honestamente siano del patrone, sì come ancho a quello tocca la spesa. V. S. avertisca di pigliar manco a credenza che può, perché col denaro in mano havrà sempre meglio conditione et non sentirà tutto di gente che li rompa il capo a chiedere o denari, $\mathrm{o}$ favore, oltra che questo è uno delli grandi honori che si possa far a un signore in quella Corte, la qual cosa quanto importi per mille rispetti V. S. lo sa. Io ho servito et conosciuto domesticamente li Reverendissimi Contarino et Polo, i quali per poveri che fossero non fecero mai debiti et ristringevano innanzi la spesa che tenere le robbe d'altri, cosa poi che finalmente piace non solo al mondo, ma a Dio.

Avertisca V. S. che la famiglia sia costumata et non diano da ragionare per Roma, come alle volte ad altri aviene, che finalmente delli scandoli si riporta sol vergogna et danno, dove del bene si dice sempre bene, et non guardate ad una corrottella indutta, parendo a molti, che come più insolenze fanno, esser di maggior conto, che questo è un abuso barbaro et indegno del nome di persone Ecclesiastiche, et massime della Casa d'un Cardinale et Maggior Penitentiero et da bene com'è il nostro. Et siate pur certo che come si costumano le famiglie, così si conservano.

So che V. S. non solo non è superba, ma amorevolissima, pure le dirò che come più sarà humana tanto più sarà amata, et come più si farà vedere agli offitiali di Casa et conversarà con loro, tanto più farà servitio al patrone et con honor suo. L'honore com'ella sa va sempre innanzi all'utile et però qui habbia avertenza, perché molti non vi guardano, anzi solo reputano honesto quello ch'è utile. Non si de far così in conto alcuno come bene ne insegna Cicerone in quei libri d'oro de officiis, l'utile sia pur il servo et l'honore il patrone, et beato quello che lo conserva in suo grado. Dice Aristotile che le bene ordinate Città sono quelle che tengono gli habitatori in unione et amore, il che voi nella vostra domestica Republica, del quale sete il console, vi ingegnarete di servare, tenendoli amorevoli insieme et al servitio del patrone, al qual voi come buon nocchiero levarete più fastidio che potrete, che a questo fine si fanno gli occonomi o maestri di Casa, per non parlar in pedantesco. Et dice il medesimo Aristotile parlando delle virtuti che la prudentia con la sapientia è com'il maior domo col patrone, il qual s'affatica et travaglia perché il signore possa più commodamente attendere a cose maggiori, sì che a voi principalmente toccano le parti della prudentia, nella quale Dio ringratiato havete gran principio et per la esperienza, con la qual'ella cresce, ne sarete ogni dì più instrutto.

Et finalmente quello ch'è il principale, anzi il tutto in questo et in ogni altro negotio ricordatevi di ricorrere a Jesù Christo signor nostro et raccomandarvi a lui di core, perché non vi abandonerà et portarà felicemente a buon fine, né dirò più di questo et forse ho detto troppo, ma datene la colpa alla richiesta vostra et all'amore che vi 
porto. Se scrivessi a qualche strano o pensassi che questa lettera havesse ad andare in mano d'altri, io sarei stato più ristrettoso et modesto, ma con V. S. non mi pare poter errare.

Resta che mi doglia seco della morte del signor suo padre et del fratello il che prima non ho inteso. V. S. conosce questo mondo, et però non si maravigliarà se tiene il suo corso, così ha fatto sempre et così farà. Contentiamoci di quello ch'a Dio piace sperando a miglior vita che questa è di nebbia, di che sono chiato afatto et la miglior mercantia che ci possiamo far è viver bene, che così a noi et alli passati avanti faremo piacere. Come V. S. scrive alla signora sua madre sia contenta consolarla et salutarla in mio nome.

A Monsignor Reverendissimo et Illustrissimo nostro signor basciarete per me la mano con quell'affetto che desidero, et caramente mi raccomandarete a tutti di Casa, et massime alli nostri conoscenti vecchi. Se l'Agathonio o il Thomasini si degnaranno scrivermi alle volte et darmi conto di loro, et di chi tiene il primo titolo di secretario, gli ne restarò con obligo. N. S. Dio V. S. et tutti conservi sani et consolati nella santa gratia sua.

Snežana Milinković

DOBRO VOĐENJE DOMAĆINSTVA. PISMO EKONOMA LODOVIKA BEKADELIJA

(Rezime)

Autorka objavljuje neizdato pismo L. Bekadelija, nadbiskupa dubrovačkog (15551560), napisano poslednje godine boravka u Dubrovniku, a upućeno mladom prijatelju L. Tedesku koji je netom postao upravitelj dvora kardinala Ranuča Farnezea. U pismu mu L. Bekadeli daje savete kako najbolje da se postavi u tom važnom i odgovornom poslu, a u kratkoj analizi koja prethodi pismu, autorka ukazuje da je i ovo skromno pismo samo dokaz želje L. Bekadelija da samoga sebe, kroz pisma, predstavi savremenicima, a nadasve potomcima, kao i da se kroz redove pisma pomalja ista ona etika koja je bila tipična za humanističke mislioce, jednog F. Gvičardinija ili B. Kastiljonea, gde su korisno i časno išli ruku pod ruku. 